\title{
THE MAǴNETIC FIELDS AND THE POLARIZATION OF RADIO EMISSION IN THE ACTIVE CENTER OF OCTOBER 1968
}

\author{
N. ERUSHEV, A. B. SEVERNY, and T. TSAP \\ Crimean Astrophysical Observatory, Nauchny, Crimea, U.S.S.R.
}

During the period of strong flare activity of the active center McMath No. 9740, from October 25 to November 2, 1968, 32 magnetograph records of longitudinal $\mathbf{H}_{\|}$ and 20 of transverse fields, $\mathrm{H}_{\perp}$, were obtained for this center with the Crimean magnetograph. Sometimes 5-7 daily records were made. $\mathrm{H} \alpha$ and $\mathrm{K}$ spectroheliograms, $\mathrm{H} \alpha$ cine film and routine determinations of sunspot magnetic fields were also obtained frequently. However the most important point is that all these optical observations were accompanied by simultaneous and continuous measurements of intensity and polarization of radio emission from this active center at a wavelength of $3 \mathrm{~cm}$, recorded with the aid of the big 22-m radiotelescope of the Crimean Observatory (the resolution is $8^{\prime}$ for this wavelength), (Erushev and Zvetkov, 1970).

The present communication contains some preliminary results about the behavior of the magnetic fields and polarization of radio emission associated with flares. Figure 1 is an example of magnetic charts before and after the flare of importance $2 n$, October 27, 1968 (14:30-16:30, Moscow time) showing very strong changes with time in the configuration of the magnetic fields. We may see in Figure 1 the disappearance of an $S$-polarity 'neck' (near $A$ ) connecting two magnetic hills of the same $(S)$ polarity $B$ and $C$, so that the hill, $A$, of $S$-polarity disappeared completely. Also very strong changes are connected with the flare of importance $2 b, 1$ November 1968, (11:01-12:03, Moscow time) as can be seen in Figure 2. We see here an enormous simplification of the magnetic structure and a decrease of gradients after the flare.

In both cases flares appear very close to or on the neutral line $\mathrm{H}_{\|}=0$ in the regions of the strongest gradients of the longitudinal field $\mathrm{H}_{\|}$, and also near the top of the big magnetic hill of $\mathrm{N}$-polarity which underwent the most rapid changes in time (cf. the similar cases in Severny and Zvereva, 1970).

From planimetry of magnetic charts of the kind we have just shown we found the total magnetic flux $F_{N}+F_{S}$ and the net flux $F_{N}-F_{S}$, correcting the corresponding area for the effects of foreshortening. Figure 3 shows the variations with time of the total flux (crosses) as compared with the plot of polarization of radio emission (dots) at $3 \mathrm{~cm}$

$$
p=\frac{{ }^{I} C^{-I} G}{I^{+I}}
$$

(at the bottom). We also plot the net flux $F_{N}-F_{S}$ in comparison with the polarization $I_{C^{-I}}$ (arbitrary units, at the top). On the horizontal axis flares of importance $>1$ are plotted. 
We observe a kind of synchronism in the behavior of magnetic fluxes and polarization of radio emission. Especially clearly pronounced is the correspondence in the decrease of fluxes $F_{N}+F_{S}$ and the decrease of polarization associated with each flare, (27, 29 October and 1 November flares). Sometimes, for instance in the cases of 28 , 29 October, even small separate fluctuations in the polarization of radio emission can be traced in corresponding changes of magnetic fluxes when magnetic measurements
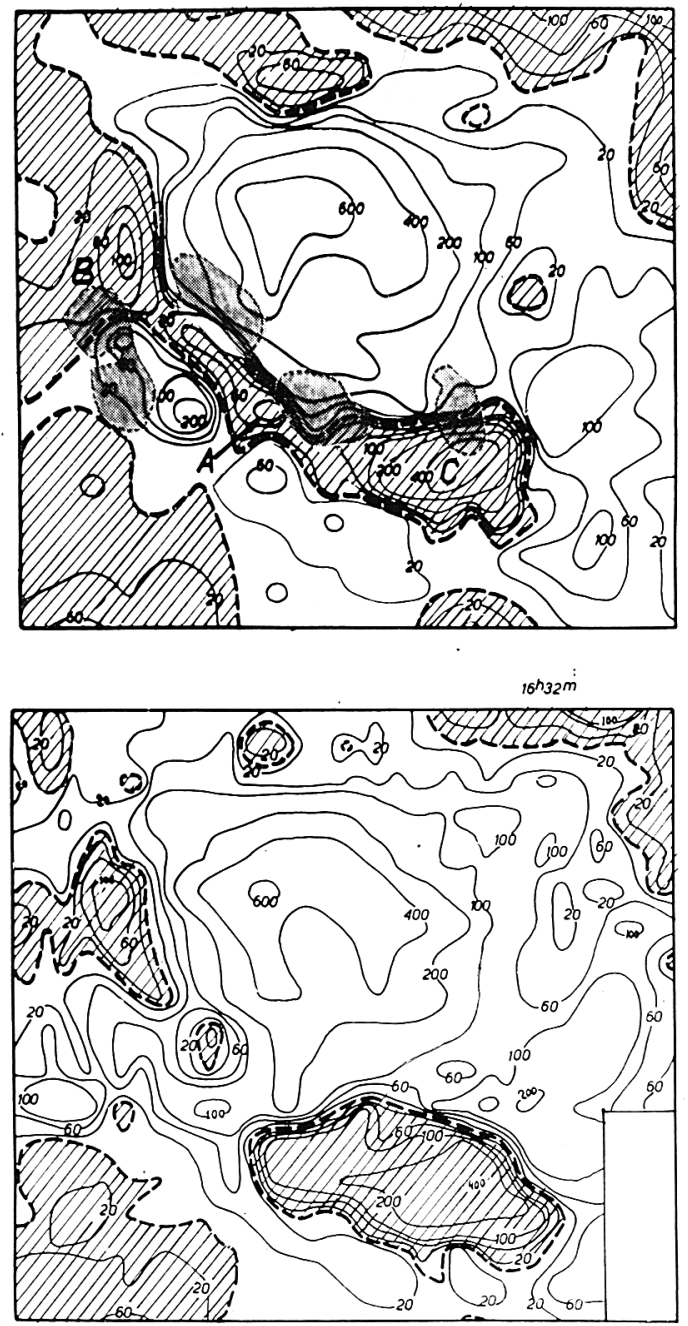

$20000 \mathrm{~km}$

Fig. 1. Showing the position of the flare 27 Oct. 1968 on magnetic map before (at the top) the flare, and the change in the pattern of magnetic field when comparing it before and after

(at the bottom) the flare. 
are frequent (the resolution in time of magnetic data is $40^{\mathrm{m}}$ in the best case). Besides this, we observe also that the general day-to-day variations are roughly the same, namely gradual increase of radio and magnetic parameters to the 29th of October and then decrease. Although the magnetic data are rather scarce, the correspondence of both magnetic and radio data can hardly be accidental.
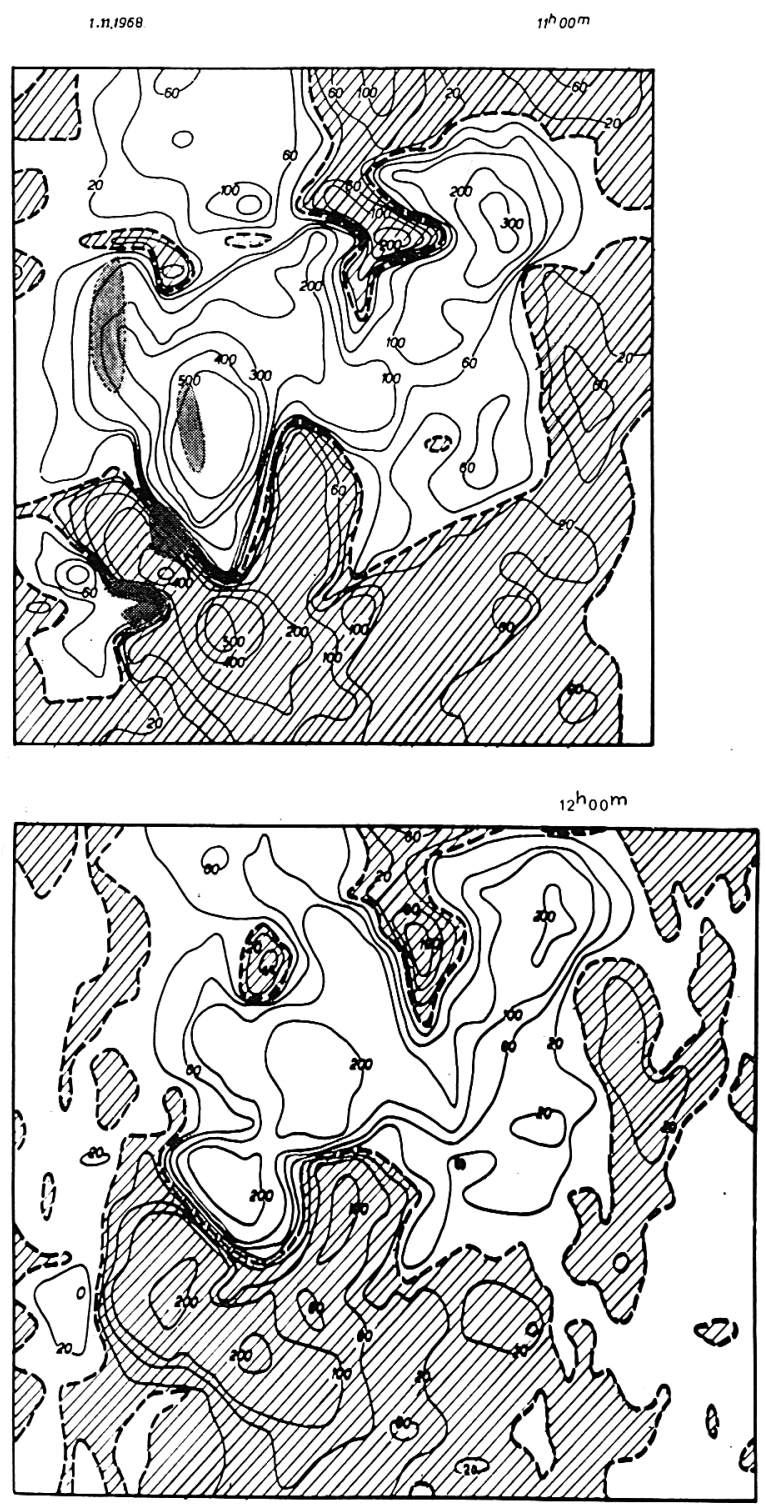

$20000 \mathrm{~km}$

Fig. 2. The same as on Figure 1 for the flare 1 November 1968. 


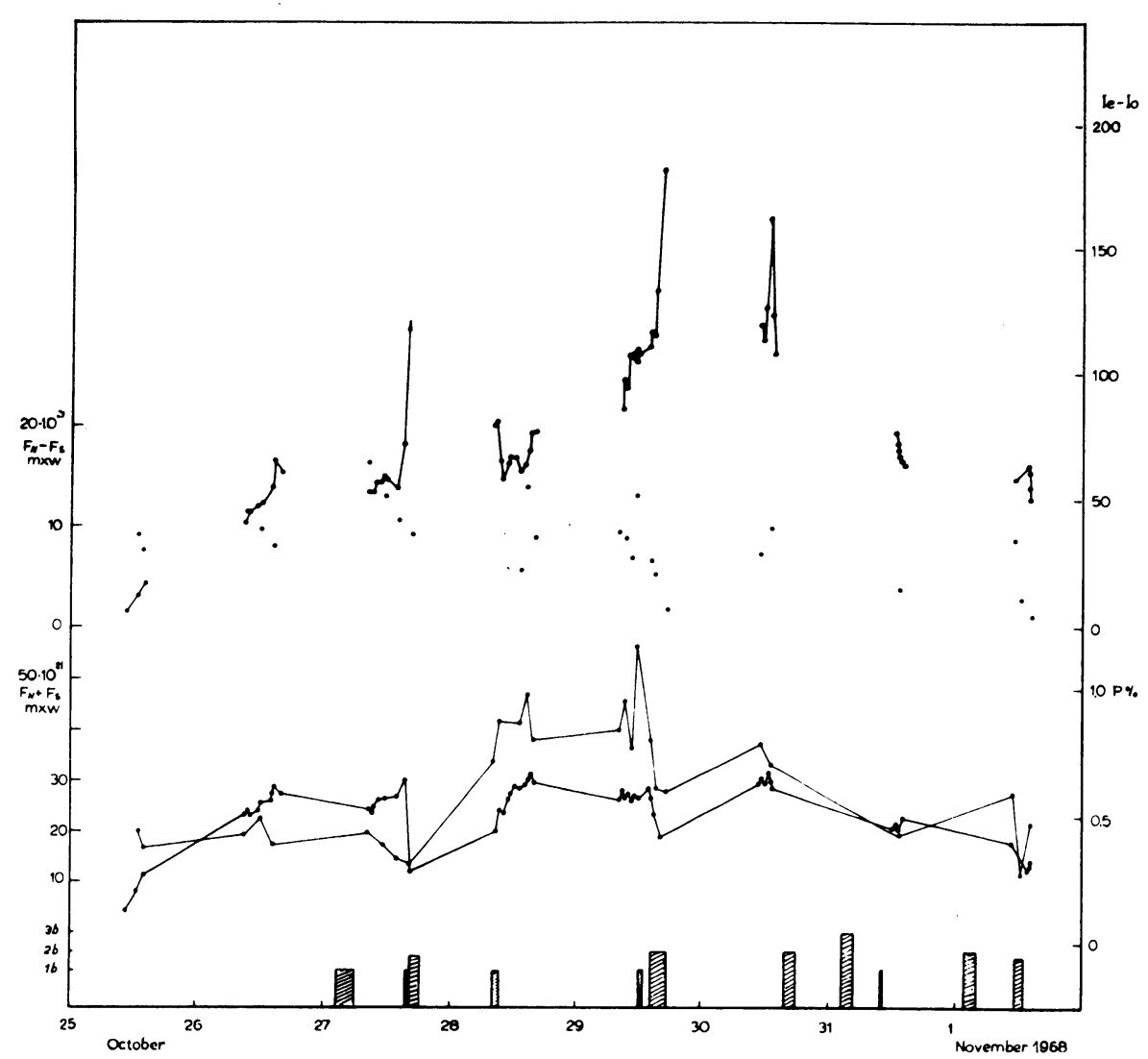

Fig. 3. The change of the percentage of polarization $P$ of radio emission at $\lambda=3 \mathrm{~cm}$ (solid dots) compared with the total magnetic flux $F_{S}+F_{N}$ (open circles, below). At the top the comparison of the polarization $I_{e}-I_{o}$ (arbitrary units) of radio emission with the net magnetic flux $F_{N}-F_{S}$.

Similarly, as in an earlier paper by Martres et al. (1968), the main change in magnetic fields is the decrease of one polarity flux. In the case considered here the other polarity flux remains practically the same, and here this was the flux of $N$-polarity of the leading spot that showed pronounced variations with time (the group was in the Southern hemisphere).

\section{References}

Erushev, N. and Zvetkov, L.: 1970, Izv. Krymsk. Astrofiz. Obs., in press.

Martres, M., Michard, R., Soru-Iscovoco, I., and Tsap, T.: Solar Phys. 5, 187.

Severny, A. and Zvereva, A.: 1970, Izv. Krvmsk. Astrofiz. Obs. 41-42, in press. 